\title{
Incommensurate Diffusion in Confined Systems
}

\author{
D. Dubbeldam, ${ }^{1}$ S. Calero, ${ }^{1}$ T. L. M. Maesen, ${ }^{2}$ and B. Smit ${ }^{1}$ \\ ${ }^{1}$ Department of Chemical Engineering, University of Amsterdam, Nieuwe Achtergracht 166, 1018 WV Amsterdam, The Netherlands \\ ${ }^{2}$ Chevron Texaco, Energy Research and Technology Center, Chevron Way 100, Richmond, California 94802-0627, USA
}

(Received 6 January 2003; published 17 June 2003)

\begin{abstract}
Molecular simulations corroborate the existence of the disputed window effect, i.e., an increase in diffusion rate by orders of magnitude when the alkane chain length increases so that the shape of the alkane is no longer commensurate with that of a zeolite cage. This window effect is shown to be characteristic for molecular sieves with pore openings that approach the diameter of the adsorbate. Furthermore, the physical compatibility between the adsorbate and the adsorbent has a direct effect on the heat of adsorption, the Henry coefficients, the activation energy, and the frequency factors.
\end{abstract}

DOI: 10.1103/PhysRevLett.90.245901

Zeolites are confined systems with pore sizes comparable to the molecular size. These microporous materials are used for separations, water softeners, and as catalysts in many (petro)chemical applications. From a scientific point of view zeolites are ideal systems to study the effect of confinement on the properties of the adsorbed molecules [1-6]. The effect of confinement on diffusion is still poorly understood despite its importance for practical applications. Experimentally this is a very difficult subject; depending on the experimental technique, the measured diffusion coefficients may differ by many orders of magnitude [7].

Zeolites are designated by three capital letter codes derived from the names of the type materials: ERI (Erionite), CHA (Chabazite), LTA (Linde Type A), OFF (Offretite), and MFI (ZSM-5 five). In 1973 Gorring reported an experimental study of diffusion of linear alkanes in ERI-type zeolite (a cage/window-type structure) as a function of chain length indicating a window effect [8]. Surprisingly, the diffusion rates reportedly increase significantly going from $\mathrm{C}_{8}$ to $\mathrm{C}_{12}$ before the usual monotonic decrease with chain length sets in. According to Gorring the diffusion rate exhibits a maximum for $\mathrm{C}_{12}$ because the shape is incommensurate with that of an ERItype cage, so that $\mathrm{C}_{12}$ is always inside an ERI-type window. Smaller molecules are commensurate with the ERI-type cage and remain trapped in its potential well.

Perhaps the simplest model for molecules that are either commensurate or incommensurate with the framework structure is the Frenkel-Kontorowa (FK) model [9] for adsorbed atoms on a periodic substrate. Models based on the FK theory have been proposed by Ruckenstein and Lee [10], Derouane, Andre, and Lucas [11], and Nitsche and Wei [12] to explain the observed increase of the diffusion coefficient as a function of the chain length qualitatively. However, due to the simplifications the quantitative predictions of these models are poor. For example, these models cannot predict the location and magnitude of the local maximum, nor can they describe the inner-cage behavior correctly. Recent attempts $[13,14]$ failed to experimentally corroborate Gorring's diffu-
PACS numbers: 66.30.-h, 47.55.Mh, 81.05.Rm, 82.75.Jn

sion data. The discrepancy between the experiments and the lack of a molecular model that quantitatively explains the window effect motivated us to develop a molecular simulation method to quantitatively study diffusion in cage/window-type geometries.

In this work we focus on self-diffusion of linear alkanes in ERI-, CHA-, and LTA-type zeolites as a function of chain length. These types of zeolites have a cage/ window-type structure with highly tortuous diffusion paths. The CHA-type cages are slightly shorter than the elongated ERI-type cages and both cage types are significantly smaller than the spherical LTA-type cages. Conventional molecular simulations are limited to relatively fast diffusing molecules $[15,16]$ or small rigid molecules [17]. Here, we combine the configurational bias Monte Carlo (CBMC) method with rare-event molecular simulation techniques $[18,19]$. The diffusion coefficients are 4-10 orders of magnitude lower than what currently can be computed by conventional molecular simulation methods.

We use the united-atom model [20] and consider the $\mathrm{CH}_{3}$ and $\mathrm{CH}_{2}$ groups as single interaction centers with their own effective potentials. The beads in the chain are connected by harmonic bonding potentials. The bond bending between three neighboring beads is modeled by a harmonic cosine bending potential and changes in the torsional angle are controlled by a RyckaertBellemans potential. The beads in a chain separated by more than three bonds interact with each other through a Lennard-Jones potential. Lattice vibrations, intergrowths and defects, cations, and associated aluminum atoms create strong disorder and complicate the diffusion process. In this work we focus on the influence of the confinement on the diffusion coefficient and therefore we use rigid all-silica ERI-, CHA-, and LTA-type molecular sieves. In all-silica structures the electric field does not vary much across the channels and cages, and Coulomb contributions to the energy of the hydrocarbons can be neglected. The chains interact with the oxygen atoms of the zeolite through a Lennard-Jones potential. Further details, including the parameters, are given by 
Vlugt, Krishna, and Smit [21] and Maesen et al. [22], who have shown that with these parameters one can reproduce the adsorption isotherms, heats of adsorption, and Henry coefficients of linear alkanes in all-silica zeolites accurately.

The structures of interest here consist of cages $A$ and $B$ separated by a narrow window forming a high free energy barrier. In the Bennet-Chandler approach $[18,19,23]$ one computes the transmission rate over the barrier in two steps. First, one computes the probability to find a chain on top of the barrier and subsequently the transmission coefficient $\kappa$, defined as the fraction of particles coming from $A$ that successfully reach $B$ out of those starting on top of the barrier. At infinite dilution the chains perform a random walk on a lattice spanned by the cage centers. The transmission rates are then easily converted to diffusion coefficients. Transition state theory (TST) assumes that no recrossing occurs $(\kappa=1)$ and predicts a transmission rate $k_{A \rightarrow B}^{\mathrm{TST}}$ given by

$$
k_{A \rightarrow B}^{\mathrm{TST}}=\kappa \sqrt{\frac{k_{B} T}{2 \pi m}} \frac{e^{-\beta F\left(q^{*}\right)}}{\int_{-\infty}^{q_{-}^{*}} e^{-\beta F(q)} \mathrm{d} q},
$$

where $k_{B}$ is the Boltzmann constant, $T$ is the temperature, $\beta=1 /\left(k_{B} T\right), m$ is the mass of the particle, and $F(q)$ is the free energy as a function of $q$. The reaction coordinate $q$ defines the progress of the diffusion event from cage $A$ to cage $B$. We define $q^{*}$ to be the location of the dividing barrier. The omission of the transmission coefficient $\kappa$ is not allowed for our systems and we correct the TST results by computing the transmission coefficients in a separate molecular dynamics calculation. It is vital to choose an appropriate reaction coordinate. By trial and error we concluded that using the second bead of the chain gave near optimal results for the systems discussed here. The transmission coefficient is $\kappa=1$ for methane and is within the range $\kappa=0.1-0.3$ for other chain lengths. The more intuitive middle bead and center of mass gave very small transmission coefficients resulting in an extremely inefficient computation.

For long chains the conventional techniques are prohibitively expensive. However, the CBMC technique used to simulate adsorption isotherms [24] can be extended to compute the free energy of a chain. In a CBMC simulation chains are grown bead by bead biasing the growth process towards energetically favorable configurations avoiding overlap with the zeolite. During the growth the Rosenbluth factor is calculated. The average Rosenbluth factor is directly related to the excess chemical potential, free energy, and the Henry coefficient [19]. The CBMC algorithm greatly improves the conformational sampling of molecules and increases the efficiency of chain insertions by many orders of magnitude. To compute the free energy as a function of the position in the zeolite, chains are inserted at random positions and grown using the CBMC scheme. The mapping of the second bead of a chain is depicted in Fig. 1. A part of the ERI-type silica

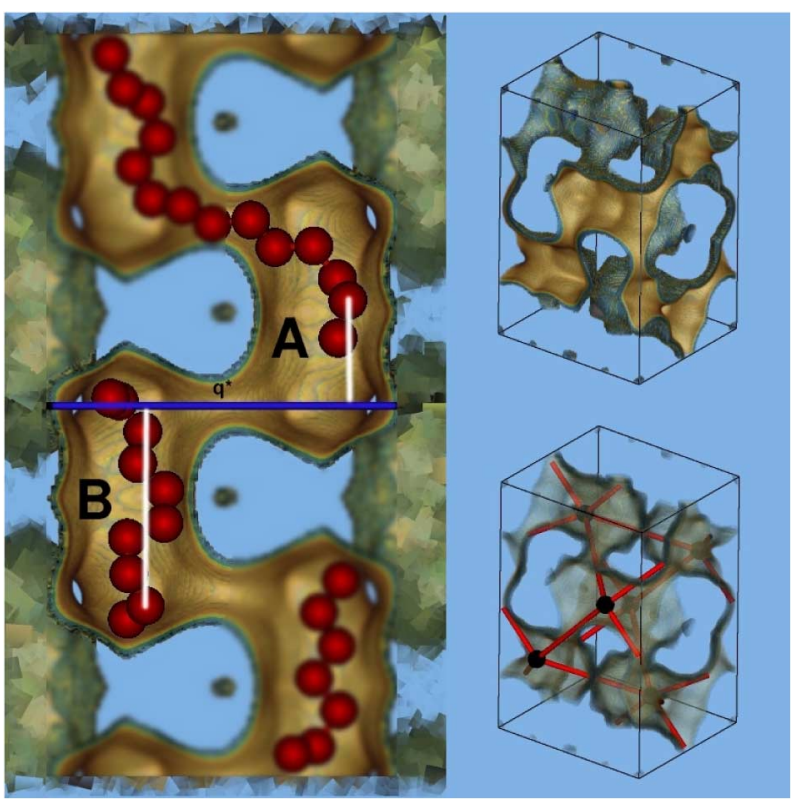

FIG. 1 (color online). The reaction coordinate $q$ is defined as the position of the second bead of a chain mapped orthogonal to the axis of projection (the line perpendicular to the window). The resulting free energy profile $F(q)$ indicates a high free energy barrier at the position of the window $q^{*}$ separating cages $A$ and $B$. On the right-hand side we show two pictures: the unit cell of ERI in 3D (top), and the connectivity of the cages within the unit cell (bottom). At infinite dilution the molecules perform a random walk on a lattice spanned by the cage centers.

with cages $A$ and $B$ (connected to other cages) is sliced half open. We show two examples: a $\mathrm{C}_{14}$ chain in cage $A$ and a $\mathrm{C}_{10}$ chain in cage $B$. Free energy values are mapped onto the one-dimensional free energy profile $F(q)$ by orthogonal projection of the position of the second bead onto the line perpendicular to the window.

For most zeolites the diffusion coefficients of linear alkanes are monotonically decreasing as a function of chain length [7]. Figure 2 shows a qualitatively different behavior in ERI- and CHA-type silica. We observe a range of intermediate chain lengths in which the diffusion coefficient is constant or increases with increasing chain length, followed by a pronounced local maximum. Good quantitative agreement with the nondisputed experimental data for LTA-type zeolite is found, although one has to realize that the scatter in the experimental data can be as much as 2 orders of magnitude. We note that the input of our simulations is the crystal structure of LTA and the force field, which has been optimized for the adsorption in MFI-type silica. It is therefore encouraging that such a good agreement is obtained without any adjustment of the parameters. More importantly, our simulation reproduced the chain length dependence and is consistent with the experimental data of Gorring [8]; we not only confirm the occurrence of a maximum for both ERI- and CHA-type zeolite at the same carbon number as observed experimentally, but also the shift of 


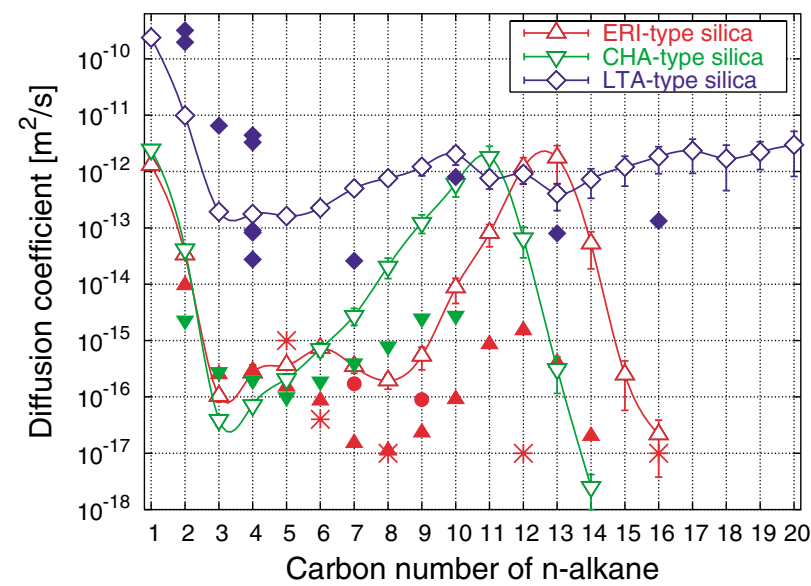

FIG. 2 (color online). Diffusion coefficients as a function of chain length at $600 \mathrm{~K}$ for ERI-, CHA-, and LTA-type zeolite; $\triangle$ ERI-type silica simulation results, $\boldsymbol{\Delta}$ experimental results of Gorring [8], * Cavalcante et al. [13], Magalhães, Laurence, and Conner [14]; $\nabla$ CHA-type silica simulation results, $\nabla$ experimental results of Gorring et al. [26]; $\diamond$ LTA-type silica simulation results,

experiment compiled in Ref. [7].

this maximum to lower carbon numbers for CHA-type silica. The increase in the diffusion rate originating from the geometry-chain interaction is a remarkable 4 orders of magnitude for both ERI- and CHA-type silicas. Additionally, we have computed the activation energies and frequency factors by fitting the diffusion results at several temperatures to the Arrhenius law. The simulation curves in Fig. 3 are qualitatively the inverse of the general shape of the diffusion curves, i.e., if a chain has a high mobility the activation energy is low and vice versa. The frequency factors (data not shown) show a maximum where the diffusion also shows a maximum and where the activation energy is at a minimum. Visual inspection of the simulation snapshots and end-to-end distance histograms show that the chain is stretched across a cage

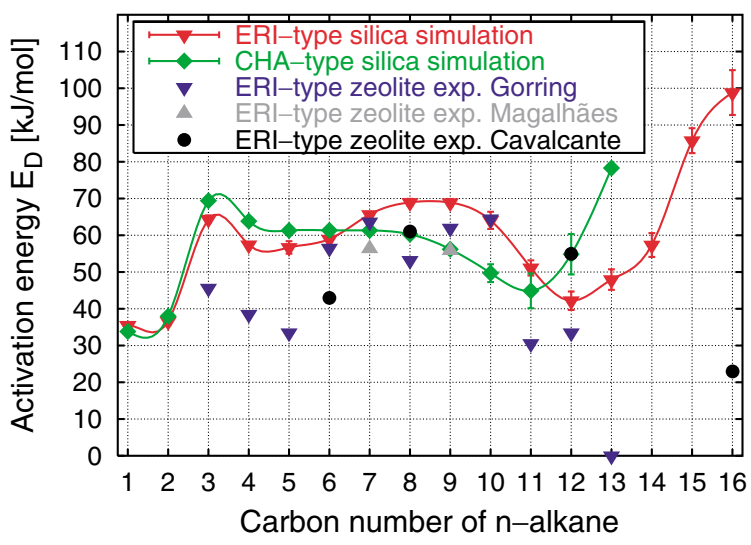

FIG. 3 (color online). Activation energies obtained from fitting diffusion simulation results at 300, 400, 500, 600, and $700 \mathrm{~K}$ to the Arrhenius law compared to the experimental results taken from Gorring [8], Cavalcante et al. [13], and Magalhães, Laurence, and Conner [14]. tethered at opposite windows: $\mathrm{C}_{13}$ for ERI-type silica, $\mathrm{C}_{11}$ for CHA-type silica, and $\mathrm{C}_{23}$ for LTA-type silica [25]. These chains correspond to the largest chains still able to fit into a single cage and have the lowest orientational freedom.

In simulations we are able to extract detailed information such as $F(q)$. The free energy differences for ERItype silica are shown in Fig. 4 and are analyzed in terms of the value inside the cage and the value at the barrier. For the chains in the cage we observe that as we increase the chain length the minimum of the free energy decreases until we reach an optimum chain length beyond which the chain no longer fits comfortably in one cage. For chain lengths longer than this optimum length the free energy increases rapidly until the chain is so big that additional beads are added comfortably in the second cage and the minimum free energy is decreasing again. For the free energy of a chain on top of the barrier we observe an increase from $\mathrm{C}_{1}$ to $\mathrm{C}_{3}$, as more beads are placed on top of the barrier. For $\mathrm{C}_{3}$ all beads feel the influence of the window. Any additional bead will be placed in more favorable positions outside the window and therefore the barrier decreases for $\mathrm{C}_{4}$ and continues to decrease until the chain is so large that it feels the limitations of the cage. Beyond this chain length the maximum of the free energy increases rapidly. Combining these effects gives the generic, nonmonotonic diffusion behavior. First a decrease followed by a possible plateau, an increase, and finally a decrease again. Key parameters in this mechanism are the presence of a narrow window combined with a cage structure. By optimizing the effective cage size one can shift the location of the second maximum to a desired value.

We have argued that the maximum of the diffusion is related to a relative unfavorable adsorption for this chain length. This is also reflected in the Henry coefficient as a function of chain length shown in Fig. 5. At sufficiently low pressures the number of adsorbed molecules is proportional to the Henry coefficient. The Henry coefficients are directly related to the minimum of the free energy

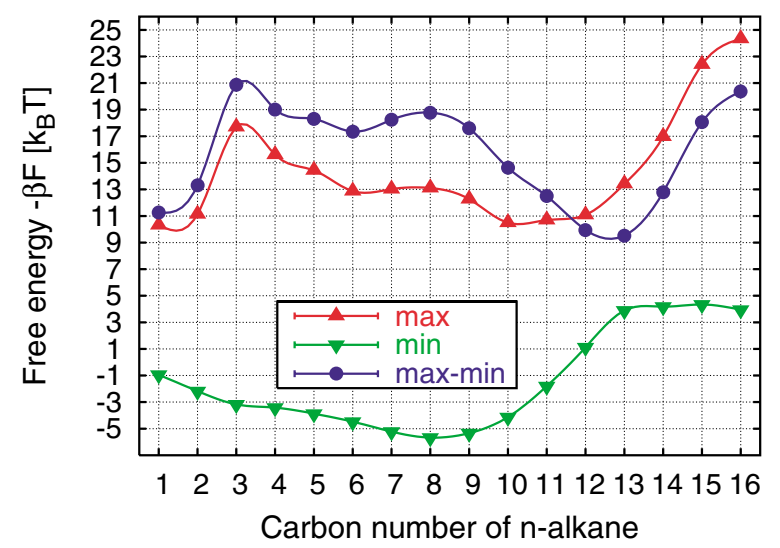

FIG. 4 (color online). The free energy difference between maximum and minimum for ERI-type silica at $600 \mathrm{~K}$. 


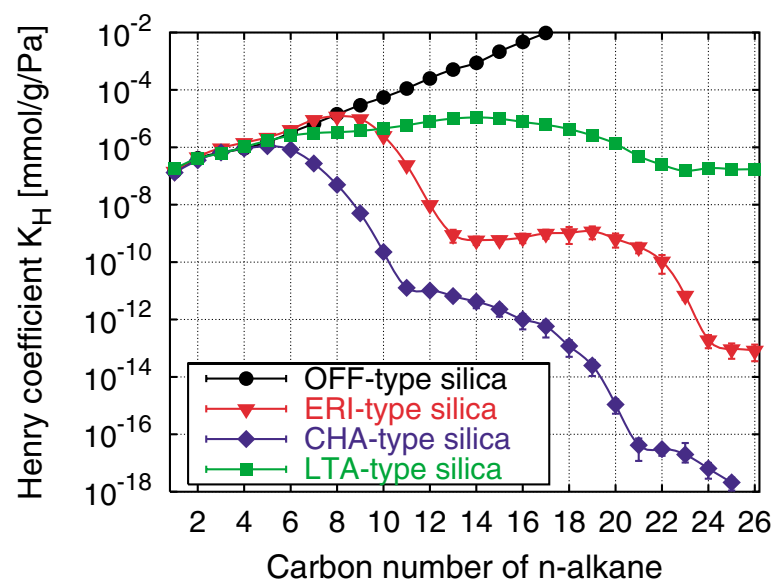

FIG. 5 (color online). Henry coefficients in OFF-, ERI-, CHA-, and LTA-type silica as a function of chain length at $600 \mathrm{~K}$.

profile. The data for OFF-type silica shows the common behavior of many channel-type zeolites. In contrast, cage/window-type zeolites show a typical nonmonotonic, periodic dependence of the Henry coefficients on the chain length. The same applies to the heat of adsorption (data not shown). The local minima nicely correspond to the local maxima in the diffusion coefficient. The repeating period in the Henry coefficients corresponds to chains fitting into one, two, or three cages, respectively. We note that the period of LTA-type zeolite is large. The confinement determines the variety of conformations present and for LTA-type zeolites this entropy effect is large as chains up to $\mathrm{C}_{23}$ still fit into a single cage. An important argument against the existence of the window effect was that experimental data for LTA do not provide evidence in support of this effect [7]. Experimentally, the maximum chain length that has been studied is $\mathrm{C}_{16}$. This chain length is in the plateau region; only for much longer chain lengths a moderate increase can be expected.

Our simulations show that the window effect is a very generic effect that can be found in an entire class of zeolites. When a chain is incommensurate with the cage structure the diffusion rate increases by orders of magnitude. The crossover points at which a chain fits in $n$ cages and a longer one into $(n+1)$ cages are directly related to the local minima in the Henry coefficients, the heats of adsorption, and the activation energies, and to the local maxima in the diffusion coefficients and the frequency factors. Although closely related, this is not necessarily an exact multiple of the window-to-window distance, nor is half the window-to-window distance the chain length which is always commensurate with the cage, as theory suggests. In fact, there can be more than one minimum of diffusion per period. From a simulation point of view it is encouraging that our method allows us to compute very low diffusion coefficients that vary 6 orders of magnitude for alkanes ranging from $\mathrm{C}_{1}$ to $\mathrm{C}_{20}$. It is particularly useful at conditions accessible to neither conventional simulation techniques nor experiments, but where diffusion limitations can be an important factor in understanding practical catalytic applications [25].

We thank the Netherlands Research Council for Chemical Sciences (CW), the European Commission, and ChevronTexaco for financial support.

[1] K. Hahn, J. Kärger, and V. Kukla, Phys. Rev. Lett. 76, 2762 (1996).

[2] H. Jobic, J. Kärger, and M. Bee, Phys. Rev. Lett. 82, 4260 (1999).

[3] L. A. Clark, G. T. Ye, and R. Q. Snurr, Phys. Rev. Lett. 84, 2893 (2000).

[4] D. S. Sholl and K. A. Fichthorn, Phys. Rev. Lett. 79, 3569 (1997).

[5] R. C. Runnebaum and E. J. Maginn, J. Phys. Chem. B 101, 6394 (1997).

[6] C. Rajappa and S. Yashonath, J. Chem. Phys. 110, 5960 (1999).

[7] J. Kärger and D. M. Ruthven, Diffusion in Zeolites and Other Microporous Solids (John Wiley \& Sons, New York, 1992).

[8] R. L. Gorring, J. Catal. 31, 13 (1973).

[9] Y. I. Frenkel and T. Kontorowa, Phys. Z. Sowjetunion 13, 1 (1938).

[10] E. Ruckenstein and P.S. Lee, Phys. Lett. A 56, 423 (1976).

[11] E. G. Derouane, J. M. Andre, and A. A. Lucas, J. Catal. 110, 58 (1988).

[12] J. M. Nitsche and J. Wei, AIChE. J. 37, 661 (1991).

[13] C. L. Cavalcante, Jr., M. Eić, D. M. Ruthven, and M. L. Occelli, Zeolites 15, 293 (1995).

[14] F. D. Magalhães, R. L. Laurence, and W. C. Conner, AIChE. J. 68, 42 (1996).

[15] E. B. Webb, G. S. Grest, and M. Mondello, J. Phys. Chem. B 103, 4949 (1999).

[16] D. Schuring, A. P. J. Jansen, and R. A. van Santen, J. Phys. Chem. B 104, 941 (2000).

[17] S. M. Auerbach, Int. Rev. Phys. Chem. 19, 155 (2000).

[18] D. Chandler, J. Chem. Phys. 68, 2959 (1978).

[19] D. Frenkel and B. Smit, Understanding Molecular Simulation (Academic, London, 2002), 2nd ed.

[20] J. P. Ryckaert and A. Bellemans, Faraday Discuss. Chem. Soc. 66, 95 (1978).

[21] T. Vlugt, R. Krishna, and B. Smit, J. Phys. Chem. B 103, 1102 (1999).

[22] T. L. M. Maesen, M. Schenk, T. J. H. Vlugt, J. P. de Jonge, and B. Smit, J. Catal. 188, 403 (1999).

[23] C. H. Bennet, in Diffusion in Solids: Recent Developments, edited by A. Nowick and J. Burton (Academic, New York, 1975), pp. 73-113.

[24] B. Smit and T. L. M. Maesen, Nature (London) 374, 42 (1995).

[25] D. Dubbeldam, S. Calero, T. L. M. Maesen, and B. Smit (to be published).

[26] N. Y. Chen, William E. Garwood, and Francis G. Dwyer, Shape Selective Catalysis in Industrial Applications (Chemical Industries, New York, 1996), 2nd ed., p. 52, Gorring's results with crystal size $r=0.3 \mu \mathrm{m}$. 